\title{
LA PROPORCIÓN ARMÓNICA EN LA ESTATUARIA DE LA CULTURA ARQUEOLÓGICA DE SAN AGUSTÍN, COLOMBIA (SOLUCIÓN FINAL PARA UN VIEJO PROBLEMA)
}

\section{DIVINE PROPORTION IN THE STATUARY OF COLOMBIA'S SAN AGUSTINN ARCHAEOLOGICAL CULTURE (RESOLVING A LONGSTANDING ISSUE)}

\section{César Augusto Velandia Jagua*}

Uno de los problemas difíciles de resolver acerca de la cultura arqueológica de San Agustín era el de cómo fueron diseñadas y construidas las esculturas funerarias que la caracterizan. El autor, luego de un largo proceso de investigación, propone una "solución final" a la vieja hipótesis de que dichas esculturas fueron concebidas dentro de un canon estético específico: la proporción armónica. Con la aplicación de un instrumento especial, el "compás de oro", demuestra cómo fue diseñada la estructura compositiva de la estatuaria con arreglo a la denominada "ley de oro" o "divina proporción".

Palabras clave: San Agustín, escultura funeraria, estética prehispánica, proporción armónica

One of the intractable problems of the San Agustin archaeological culture is how funerary sculptures were designed and constructed. As a result of an extensive investigation, the author proposes a "final resolution" to the longstanding theory that these sculptures were conceived within a specific aesthetic canon-the harmonic proportion. By applying a special instrument, the "golden compass," the author demonstrates how the statues' structural composition was designed in accordance with the so-called "golden rule" or "divine proportion."

Key words: San Agustín, funerary sculpture, pre-Hispanic aesthetics, harmonic proportion

\section{ESPACIO Y TIEMPO DE LA CULTURA DE SAN AGUSTIIN}

Los restos de la cultura arqueológica de San Agustín se encuentran dispersos en un extenso paisaje, cuyo centro es un gran macizo montañoso de la cordillera de los Andes, al sur de Colombia. Dicho territorio colinda al oeste con la selva aledaña al océano Pacífico, al sur con la cordillera andina y al este con las selvas de la gran cuenca superior del río Amazonas. La mayor concentración de materiales arqueológicos se halla en la jurisdicción de los municipios de San Agustín e Isnos, departamento del Huila, y de Pisimbalá e Inzá (Tierradentro), en el departamento del Cauca; que comprenden la región meridional de la cuenca alta del río Magdalena.

En una época se pensó que la cultura de San Agustín era un hecho aislado que, paradójicamente, tendría más relaciones con las altas culturas americanas (Chavín de Huántar, Recuay, Olmeca, etc.) que con las culturas prehispánicas más próximas en Colombia. Esto se debía al relativo desarrollo de los estudios arqueológicos y, por tanto, al desconocimiento que teníamos de estas culturas. Hoy pensamos diferente: la llamada cultura de Tierradentro, por ejemplo, es un desarrollo regional de San Agustín y culturas que se consideraban lejanas, como el complejo Calima (Ilama, Yotoco y Sonso), situadas en el valle del río Cauca, están más próximas de lo que

\footnotetext{
* César Velandia, director Grupo arqueo.región (Grupo de Investigación en Arqueología, Patrimonio y Ambiente Regionales), Facultad de Ciencias Humanas y Artes, Universidad del Tolima, Ibagué, Colombia, email: velandia@ut.edu.co
} 
antes estimábamos; estas relaciones las encontramos en la cerámica y la iconografía de la orfebrería en oro y cobre, así como en las prácticas funerarias y los enterramientos en sarcófagos de madera.

La cultura de San Agustín ha sido caracterizada por sus grandes construcciones funerarias megalíticas y sobre todo por la estatuaria, a la que se ha dedicado relativamente la mayor atención por parte de los investigadores. Pero en los últimos años, y mediante el planteamiento de nuevos enfoques en los problemas de la arqueología regional, los trabajos han apuntado con mayor énfasis en la reconstrucción de las pautas o patrones de asentamiento mediante exploraciones sistemáticas extensivas y dataciones absolutas. El resultado inmediato ha sido la ampliación de las áreas de trabajo y el establecimiento de correlaciones más objetivas con las culturas americanas. Además se ha desarrollado un interés en las estructuras económicas y organizativas de las sociedades prehispánicas, en particular hacia los cacicazgos o señoríos, que se presumen como la estructura fundamental de la sociedad de San Agustín.

A pesar del énfasis puesto antaño en la estatuaria, esta no ha sido correlacionada temporalmente en términos de datación absoluta, pues la mayor parte de las construcciones monumentales fueron objeto del saqueo y la depredación, aun desde tiempos anteriores a la conquista europea. Sin embargo, las excavaciones de las últimas décadas, controladas científicamente, y el consiguiente fechado del material funerario en que se ha encontrado la estatuaria, permiten consolidar algunos lapsos del proceso que, desde una perspectiva relativa, había sido clasificado en tres grandes períodos en el contexto del desarrollo regional del suroccidente de Colombia: Formativo (1000-300/200 AC); Clásico Regional (300/200 AC y 900/1300 DC) y Período Tardío o Reciente (500-1500 DC) (Rodríguez 2005) (fig. 1).

\section{ANTECEDENTES DEL PROBLEMA}

Desde el comienzo de la investigación relativa a la iconografía en la estatuaria y la parafernalia funébricas de San Agustín, asumí, como referente obligado de las múltiples preguntas que se iban abriendo paso, el reto de resolver la descripción formal de las esculturas y de explicar de qué manera fueron diseñadas, lo que equivale a prefigurar de qué manera fueron pensadas o imaginadas. El antecedente mejor ilustrado para esa época, inicios de la década de 1980, lo tenía en la obra del maestro Luis Ángel Rengifo, quien desde la perspectiva de su oficio como pintor y grabador, amén de su calidad académica como profesor e investigador en la Facultad de Bellas Artes de la Universidad Nacional de Colombia, planteó una tesis según la cual la estatuaria de San Agustín habría sido concebida y realizada a partir de un canon geométrico particular, denominado como la "proporción armónica", "divina proporción" o "número de oro" (Rengifo 1962, 1966). ${ }^{1}$

Si bien en aquellos trabajos de los ochenta el interés central se enfocaba en el cómo de las estructuras, ahora las preguntas van un tanto más allá, hacia la explicación de cómo fueron diseñadas y construidas; en el primer caso, se trataba de explorar la estructura de lo representado, ahora se trata de explicar un modelo de la estructura de las representaciones. Para este propósito es indispensable retomar los tramos avanzados por Rengifo, de cuyo trabajo se desprenden tres criterios importantes: a) la estatuaria obedece a una composición geométrica específica; b) el concepto básico de la composición es planigráfico, y c) los elementos compositivos esenciales son el rectángulo y el cuadrado.

A partir de estas características ortogonales de la estatuaria y de las ideas del maestro Rengifo, propuse (Velandia 1994: 43, 1999: 194, 2006: 238) algunas opciones explicativas acerca de la composición de los elementos icónicos en la estatuaria, pero hasta ahí llegó mi exploración; pues el trabajo estaba centrado en la iconografía propiamente dicha. Ahora intentaré correr un poco la explicación hacia adelante. Para este momento, puedo contar con otras referencias investigativas respecto de la iconografía en escultura prehispánica ${ }^{2}$ y en particular con un modelo analítico acerca de la geometría de Mesoamérica, de Margarita Martínez del Sobral (2000), en el que se expone la mejor argumentación y demostración de que las expresiones estéticas en las sociedades prehispánicas -por lo menos para Mesoamérica- se rigieron por un canon geométrico específico que se hallaba fundamentado en la comprensión de un concepto geométrico complejo, que en Occidente fue denominado como la "proporción armónica".

El arte mesoamericano no fue resultado del azar o del gusto del artista, sino que obedeció a principios geométricos aplicados consistentemente. Las líneas, los círculos, los cuadrados y los rectángulos se combinaron armónicamente y conformaron las bases en las que se desarrollaron el arte y la arquitectura precolombinos (Martínez del Sobral 2000: 11).

Sin embargo, al consultar la información en el registro arqueológico de otros lugares de América, parece posible que dicho rigor y tales ordenamientos del arte mesoamericano también fueran desarrollados por otras sociedades prehispánicas. 


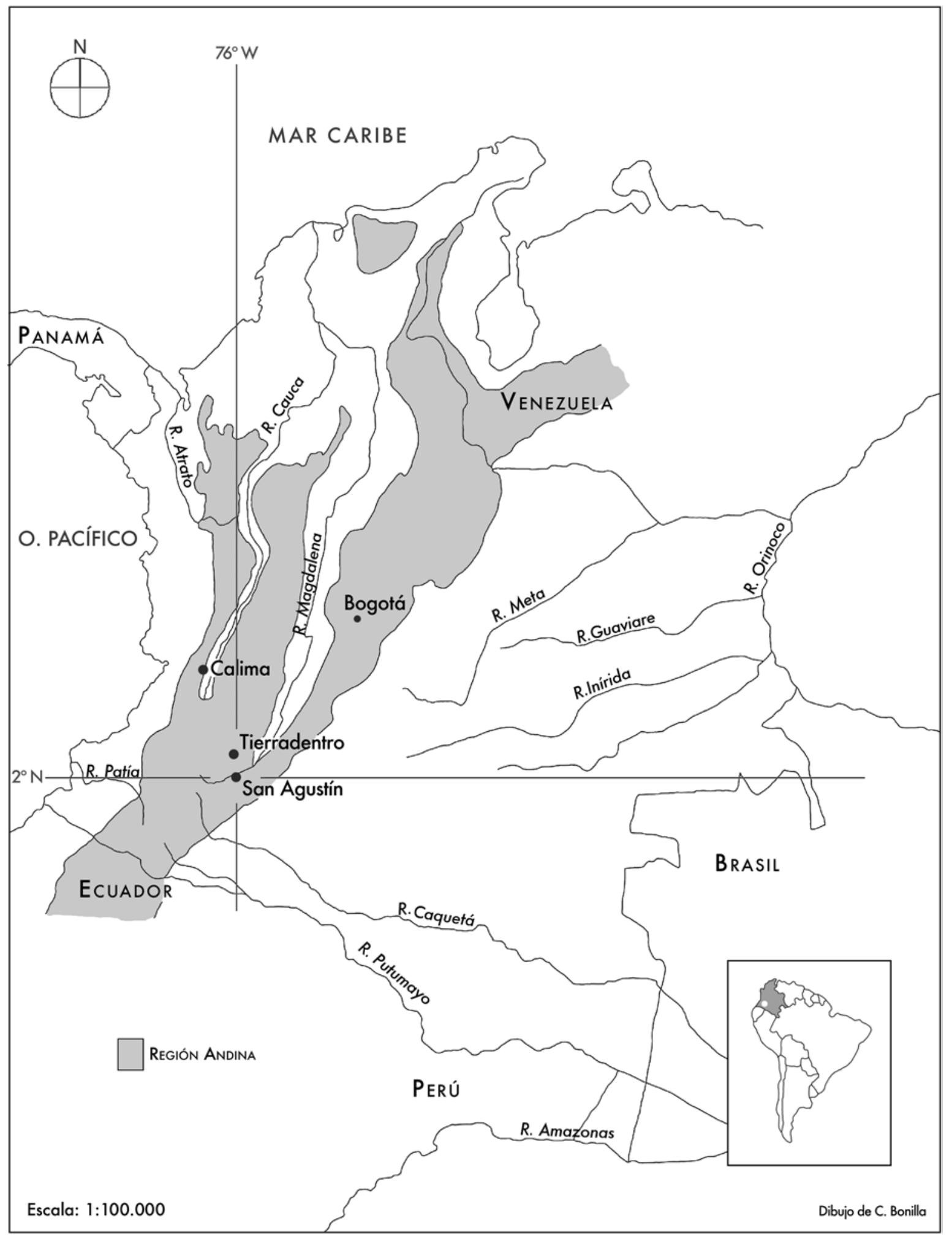

Figura 1. Ubicación geográfica del registro arqueológico de San Agustín. Figure 1. Geographic location of the San Agustín archaeological record. 


\section{DE LA PROPORCIÓN ARMÓNICA}

A diferencia de la situación en Mesoamérica, donde la información del registro arqueológico tiene el correlato de la invaluable (aunque escasa) información de los códices, el caso que nos ocupa con la estatuaria de San Agustín tiene el inconveniente de que la información contextual no existe, ya que la mayor parte de los sitios funerarios en que se halló fueron saqueados, tal vez desde tiempos anteriores a la conquista, como ya se dijo. Otra circunstancia estriba en que en San Agustín no existe propiamente arquitectura edilicia, aparte de las construcciones funerarias de carácter megalítico. No obstante, de la información inherente al arte escultórico es posible derivar la suficiente información para intentar, por lo menos, una descripción formal.

El particular interés por la iconografía tiene implícita la pregunta por su construcción y, aunque era posible aislar los elementos icónicos, siempre estuvo en el aire la dificultad para contextualizar geométricamente las formas escultóricas. Para el análisis de la estructura de su composición trataré, en lo posible, partir de la información empírica disponible, esto es, desde las formas en la estatuaria, con el fin de controlar el modelo analítico. Por tanto, en lugar de enunciar la teoría de un posible esquema para aplicarlo luego como horma o patrón a la estatuaria -que tendría el riesgo de hacer ver lo que necesariamente no está ahí-, iniciaré la descripción desde las formas geométricas deducibles en la estatuaria y, al mismo tiempo, iré explicando de qué manera se construye una cierta proporcionalidad en la construcción y la articulación de las partes respecto del todo de las estructuras.

El maestro Rengifo emprendió una explicación desde el enunciado del concepto de la "proporción armónica" y mediante la aplicación del "rectángulo dorado" intentó descifrar, en varios casos escogidos al efecto, la estructura geométrica implícita en cada escultura. Héctor Llanos (1995: 76), siguiendo algunos conceptos de Rengifo, en particular que la estatuaria es planigráfica, argumentó que:

Las diferentes partes de la escultura fueron medidas con un patrón preciso, que pudo ser un cordel o una vara similar a nuestro metro, aunque de magnitud diferente. Aunque este patrón de medida parece haber tenido una representación geométrica (bidimensional), el cuadrado y el paralelogramo. El cruce de las dos diagonales de estas figuras se ubica en el centro, que en el plano del rostro se ubica en la mitad de la nariz, en el plano del tronco y las piernas en la mitad de ellos. El eje central de una escultura pasa por estos tres puntos centrales.

Esta deducción es casi tautológica, ya que el trazado de las diagonales solo corrobora que el plano es un paralelogramo, pero no define la estructura de la escultura. De otra parte, si un rostro se inscribe en un cuadrado, el cruce de las diagonales estará en el centro de la nariz simplemente porque la nariz está en la mitad del rostro, pero no tiene nada que ver con la estructura del cuadrado. De alguna manera, esta fue también la dificultad del maestro Rengifo cuando intentó demostrar la estructura del "rectángulo dorado" como la correspondencia de una plantilla geométrica sobre cada escultura. Y también fue mi dificultad durante mucho tiempo, porque estaba persuadido -siguiendo el criterio del maestro- de poder interpretar la estatuaria con un modelo geométrico a la mano.

El estudio de la obra de Martínez del Sobral (2000) fue esclarecedor en muchos aspectos, no solo por su rigor conceptual, sino por su interés metodológico en el análisis iconográfico. A este respecto plantea, como un recurso indispensable, la existencia de un "compás áureo" o "compás de oro", al que considera "[...] de suma utilidad en el análisis geométrico, ya que permite saber si las figuras analizadas contienen o no dicha proporción, de inmediato y con una sola medición" (Martínez del Sobral 2000: 22); del que realizó una reconstrucción a partir de un diseño del arquitecto Carlos Chanfón, porque "nunca he encontrado compás como este en el mercado, ni en América ni en Europa. Es un instrumento que seguramente fue utilizado en la Edad Media, ya que la Divina Proporción fue profusamente usada en esa época, sobre todo en la arquitectura gótica" (Martínez del Sobral 2000: 22).

Persuadido de las bondades de la internet y luego de una prolija búsqueda, encontré no solo información más amplia al respecto, sino también que en el pueblito de Auvillar, cerca de Lyon, Francia, había un taller especializado en la fabricación de armas medievales e instrumentos antiguos y un catálogo en que se ofrecía a la venta un compas d'or. Luego de los contactos adecuados y de hacer una detenida explicación de para qué lo requería, M. Robert Losson, el artesano fabricante, me envió en calidad de donación un ejemplar del "compás de oro"... en acero inoxidable (fig. 2). ${ }^{3}$

Siguiendo las instrucciones de Martínez del Sobral (2000), encontré rápidamente una solución para establecer un procedimiento analítico de la estatuaria, de la que se presumía que estaba construida según el canon de la "divina proporción". Pero antes de proceder al análisis de la "divina geometría" en la estatuaria de San Agustín, debo hacer una sucinta explicación acerca de la "proporción armónica".

Este concepto tiene una vieja historia, pues fue descrito por Pitágoras y Euclides y aplicado en el diseño de las antiguas arquitecturas egipcia, griega, china, etc., y en 


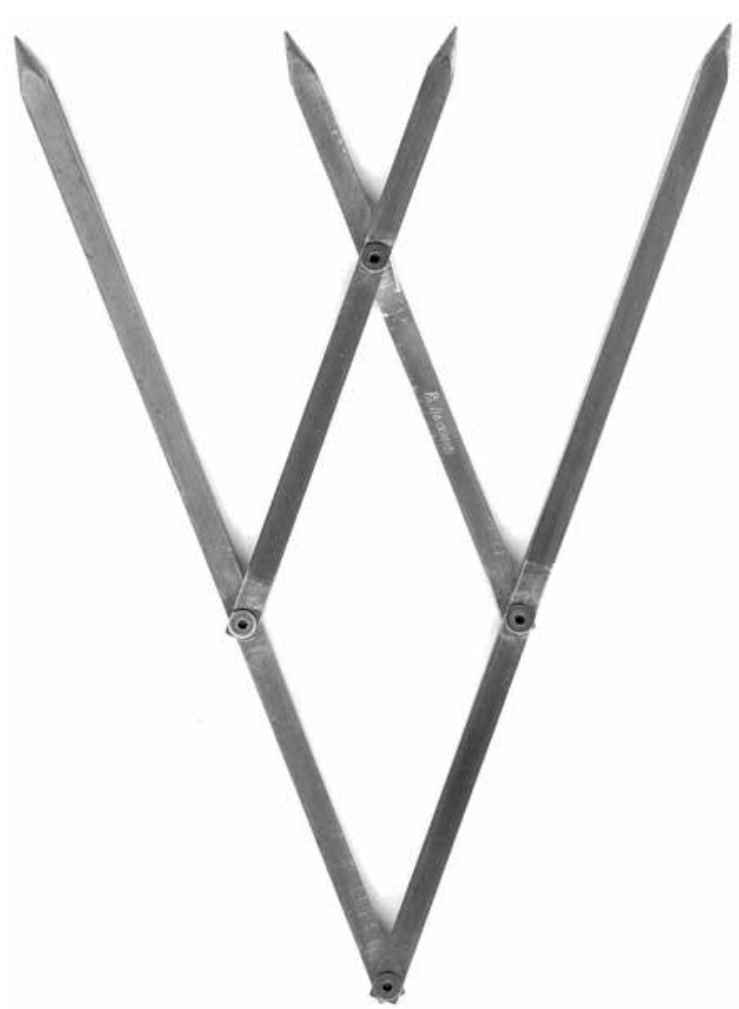

Figura 2. El "compás de oro".

Figure 2. The "golden compass."

las representaciones plásticas de casi todas las culturas del mediterráneo europeo. Su enunciado formal se debe a fray Luca Pacioli di Borgo quien publicó en Venecia, en 1509, el tratado De divina proportione (Ghyka 1953: 9) con ilustraciones de Leonardo da Vinci (Pacioli di Borgo 1991).

La "proporción armónica" tuvo decisiva influencia en todos los pintores, escultores, astrónomos y arquitectos del Renacimiento y se encuentra, por medio de ellos y como parte de la herencia helenística, en la base de nuestro canon estético. El hecho de su presencia casi universal se debe no tanto a que el mundo hubiera sido civilizado por la antigua Europa, sino a que esta relación se halla en la naturaleza, en los procesos y los ritmos de crecimiento de los seres vivos, tanto vegetales como animales, así como en la composición de los cristales. Debido a que la naturaleza es la misma en todas partes, los estudiosos de diversas sociedades -tanto del Viejo Mundo como de América- dedujeron de su observación un concepto geométrico y, por tanto, estético, que se convirtió en canon o norma para la construcción de las expresiones plásticas y la arquitectura en diversas culturas (Ghyka 1953: 119; Sampaolesi 2006: 9; Hemenway 2008: 123; Corbalán 2009: 118; Orozpe 2010: 48).
La "proporción armónica" es el resultado de establecer una relación entre dos formas geométricas elementales, como la generada por la partición de un segmento de línea en dos partes desiguales, o entre dos formas distintas como el cuadrado y el rectángulo, relación en la cual "la parte menor es a la mayor, como esta a la suma de las dos", lo que se expresa de esta manera:

$\mathrm{a}: \mathrm{b}:: \mathrm{b}: \mathrm{c}$, donde $\mathrm{c}=(\mathrm{a}+\mathrm{b})$

$a: b:: b:(a+b)$

Este modo de la relación entre tres términos $(\mathrm{a}: \mathrm{b}$ : c), donde el tercero es igual a la suma de los dos primeros, es única en la geometría y, tanto por este hecho como por el equilibrio y la economía conceptual que plantea, se le denominó desde los tiempos de Pitágoras como "sublime" y "armónica"; hasta el Renacimiento cuando fray Luca Pacioli la definió como "divina proporción". La relación entre los términos se expresa en un número (1.618), que en 1924 fue denominado por Theodore Cook con la letra $\phi$ (Fi, por Fidias el escultor y arquitecto griego) (Sampaolesi 2006: 15). Dicho número se conoce también como el "número de oro" según Leonardo da Vinci, o "sección áurea”, según lo definió el matemático y astrónomo Johann Kepler en 1596, en su Prodromus dissertationum cosmographicarum. La relación entre el cuadrado y el rectángulo, que genera un rectángulo que es la suma de los dos, se denomina como "rectángulo dorado".

La "sección áurea" es el resultado de dividir una longitud en dos partes desiguales, de tal manera que entre la parte menor y la mayor exista la misma razón que entre la parte mayor y el todo. Para encontrar la relación se traza una línea, $X Y$, en cuyo extremo se levanta la perpendicular YZ. Luego se divide XY en dos partes iguales, y con esta medida se hace centro en $\mathrm{Y}$, para obtener el punto Z. Ahora se unen los puntos XZ. Utilizando el compás, con centro en $Z$ y con la medida ZY se traza un arco que corte la hipotenusa en el punto E. Haciendo centro en X, y con la medida XE, se traza un arco que corte la línea XY en el punto N. De este modo, la línea XY ( c ) queda dividida en dos segmentos desiguales XN ( a ) y NY ( b ) (fig. 3).

A partir de un cuadrado y con centro en $m$ (punto medio del lado $\mathrm{AB}$ ) se traza un arco que corte la prolongación de la base del cuadrado $\mathrm{AB}$ en el punto $\mathrm{C}$, en el cual se levanta una perpendicular hasta la altura del cuadrado. Se observa que el movimiento de la diagonal ha generado un rectángulo (de base BC) que, sumado al cuadrado (de base $\mathrm{AB}$ ), produce a su vez un rectángulo mayor (de base a) que tiene la misma proporción del rectángulo menor (fig. 4). 


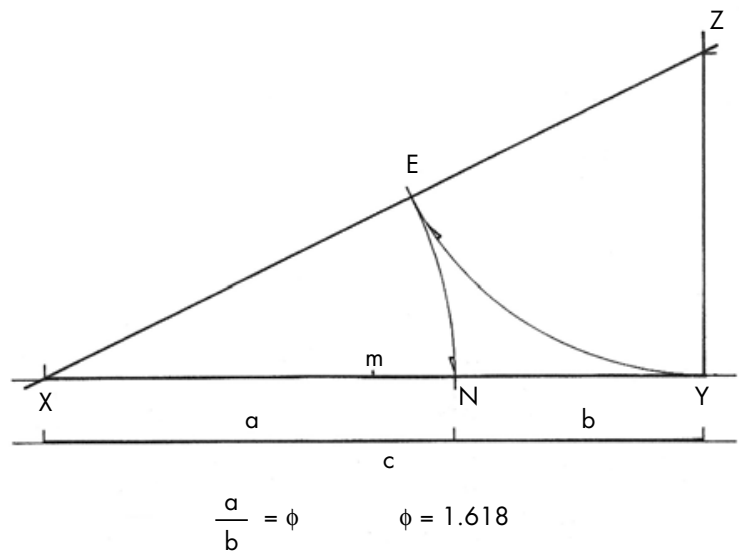

Figura 3. Definición de la "sección áurea". Figure 3. Definition of the "golden section."

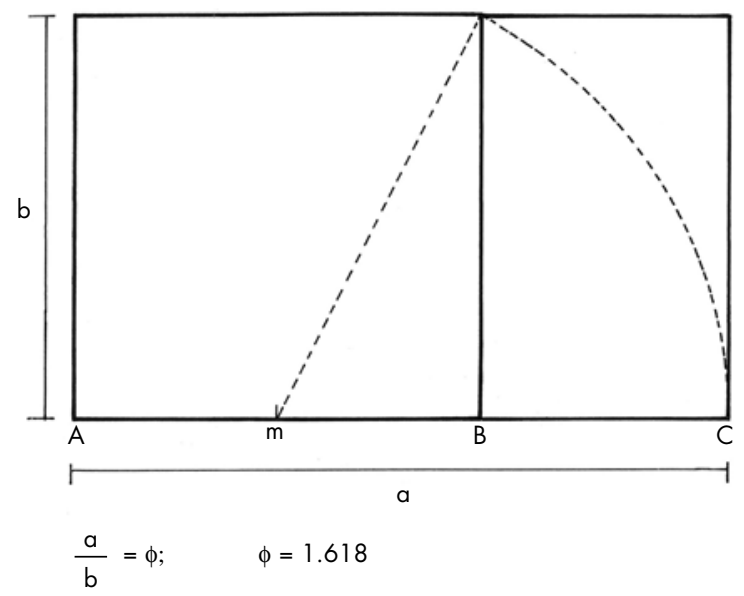

Figura 4. Construcción del "rectángulo dorado".

Figure 4. Construction of the "golden rectangle."

La proporcionalidad del "compás de oro" está determinada no solo por su estructura peculiar, sino por la relación de sus dimensiones. En el caso de este ejemplar, que está compuesto por dos piezas largas y dos cortas, las patas largas están divididas en dos segmentos de 8 y 13 centímetros, y las cortas, en dos segmentos de 5 y 8 centímetros. Abierto, en su máxima extensión, abarca tres valores de 13, 21 y 34 centímetros (fig. 5).

La relación entre estas dimensiones establece una serie numérica:

$$
58132134
$$

Dicha serie se conoce como "sucesión de Fibonacci" y fue enunciada por Leonardo de Pisa en 1202. En dicha serie, cada número es igual a la suma de los dos anteriores:

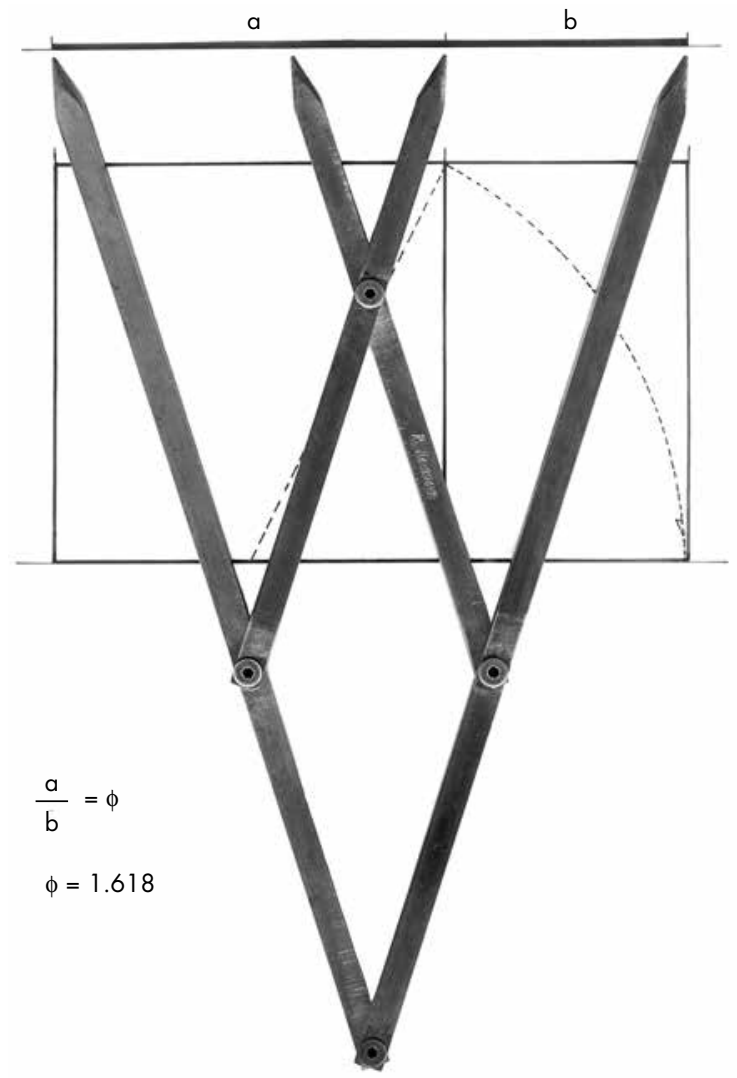

Figura 5. El "compás de oro" y la "proporción armónica". Figure 5. The "golden compass" and the "harmonic proportion."

\section{3 , etcétera.}

Pero al dividir cada número por el anterior, a partir del quebrado $34 / 21$, se obtiene como resultado un número constante, 1.618, el "número de oro".

La particularidad de esta relación, entre el cuadrado y el rectángulo, en la cual una forma (el cuadrado) determina la formación de una forma distinta (el rectángulo), la que sumada a la primera constituye una estructura que tiene la misma proporción que el segmento adicionado se puede observar mejor en un gráfico (fig. 6).

\section{LA PROPORCIÓN ARMÓNICA EN SAN AGUSTIN}

Ahora procederé a la inspección mediante el "compás áureo" de algunos ejemplares de la estatuaria de San Agustín. Pero antes de intentar alguna explicación, debo mostrar cómo opera el "compás de oro". Para esto, he seleccionado una pieza ubicada en la Mesita C del Parque Arqueológico de San Agustín, distinguida con el código 

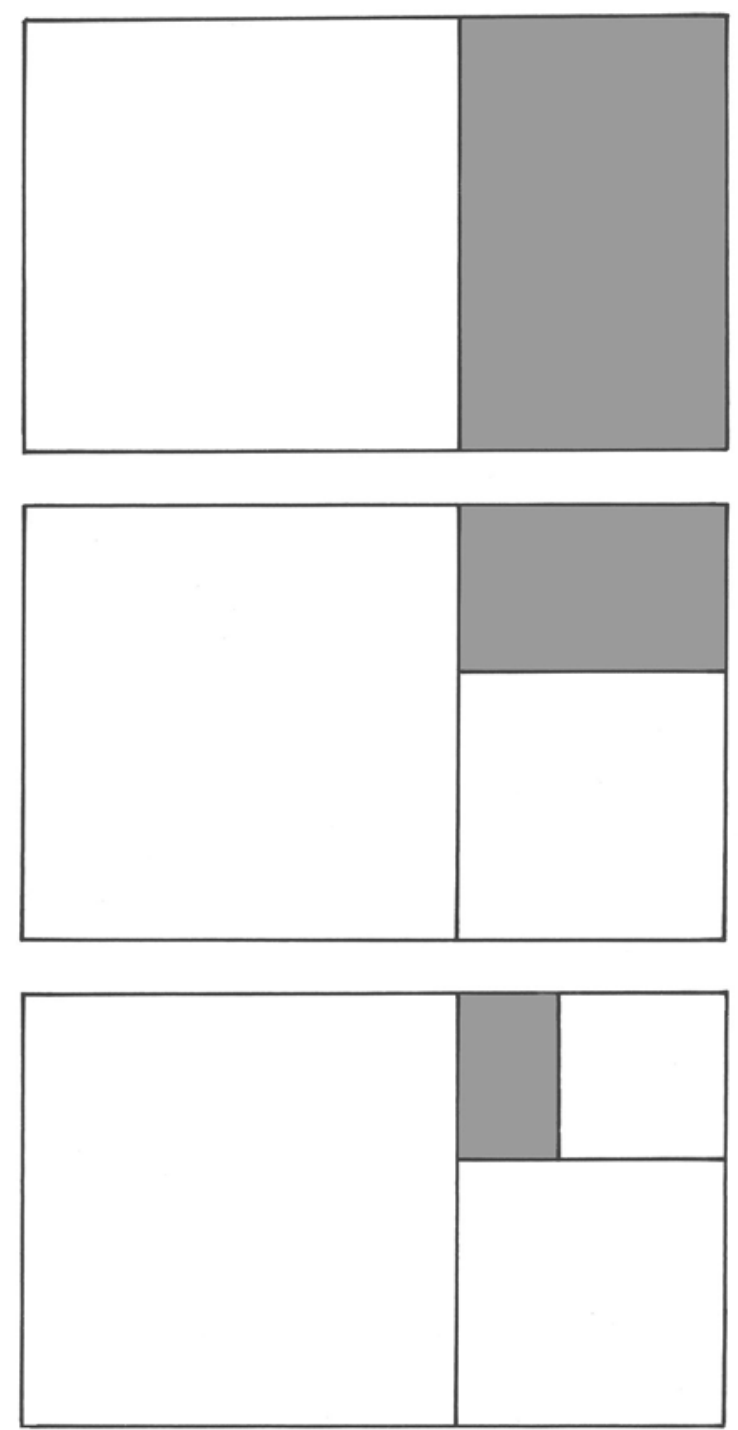

Figura 6. Subdivisión sucesiva de cuadrados y rectángulos en "proporción armónica".

Figure 6. Successive subdivision of squares and rectangles in "barmonic proportion."

MC-109. Esta escultura tiene la peculiaridad de que no fue terminada, pues el trabajo se suspendió apenas se cumplía la etapa de boceto y se había comenzado a diferenciar algunas zonas y planos para construir la representación de un personaje enmascarado que sostiene un instrumento entre las manos (fig. 7).

Como ya he planteado, de las esculturas de San Agustín se enfatizó su carácter planigráfico y que, aparentemente, estuvieran proyectadas dentro de una estructura ortogonal. Dentro de varias piezas consideradas en particular, destaca esta escultura porque muestra algunos detalles de su proceso de elaboración, como que está dividida en dos grandes planos mediante una línea vertical trazada por el centro de la estructura (que, luego en el proceso del trabajo debía desaparecer), lo que establece una relación de analogía formal entre los elementos inscritos en cada plano. Esta relación denominada "simetría bilateral" se debe en lo fundamental al sentido de lo representado: un cuerpo natural que, de hecho, está estructurado simétricamente (Washburn \& Crowe 1988: 14; Weil 1990: 20; Kubovy \& Strother 2004: 23).

Considerando que la presencia del esbozo de línea vertical $(\mathrm{AD})$ era la demostración de la presunción de que la proyección del diseño era ortogonal, abrí el "compás de oro" a lo largo de la misma tratando de hacer coincidir puntos específicos con las cuatro puntas del mismo (fig. 8).

Sobre el dibujo que había elaborado a escala, coincidían tres puntos sobre el área visible de la pieza: A, B y C; el punto D queda por debajo de la superficie del prado en que se encuentra erigida. ${ }^{4}$ Una vez identificadas estas magnitudes, procedí a deducir los planos determinados por estos referentes de proporción; así, levanté un cuadrado a partir del segmento mayor del compás, AB, y desde el punto medio del lado del cuadrado, tracé un arco hasta cortar la prolongación de la base del cuadrado, para generar el lado menor del rectángulo complementario. De esta forma aparece un rectángulo dorado formado por la suma del cuadrado (a) y el rectángulo (b). Al aplicar el mismo procedimiento sobre la zona inferior de la pieza, aparece entonces que el rectángulo (b) es también el complementario del cuadrado (c) para formar un rectángulo dorado inverso (fig. 9).

Este juego de relaciones se establece a cada lado de la línea divisoria de manera exacta, de tal modo que en el lado opuesto (derecha del observador) aparece la misma correlación geométrica. Sobre la zona superior, que conforma la máscara del personaje, tenemos que los dos cuadrados simétricos se subdividen en cuatro cuadrados adyacentes (d, e, f y g) (fig. 10) y, al trazar sus respectivas diagonales, estas determinan unos puntos específicos necesarios para la determinación, tanto de la distribución de los espacios como de los elementos propiamente figurativos como los ojos, la boca y la máscara (fig. 10).

La intersección de las diagonales, en h1, h2 y h4, determina los puntos desde los cuales se trazan los semicírculos que definen la forma externa de la máscara. El punto h3, desde el centro de la nariz, define tanto la altura y el punto inferior de la máscara como los referentes externos de los ojos (fig. 11). En el siguiente caso (fig. 12) tenemos una de las esculturas más conocidas de San Agustín que, a pesar de su ortogonalidad, siempre 

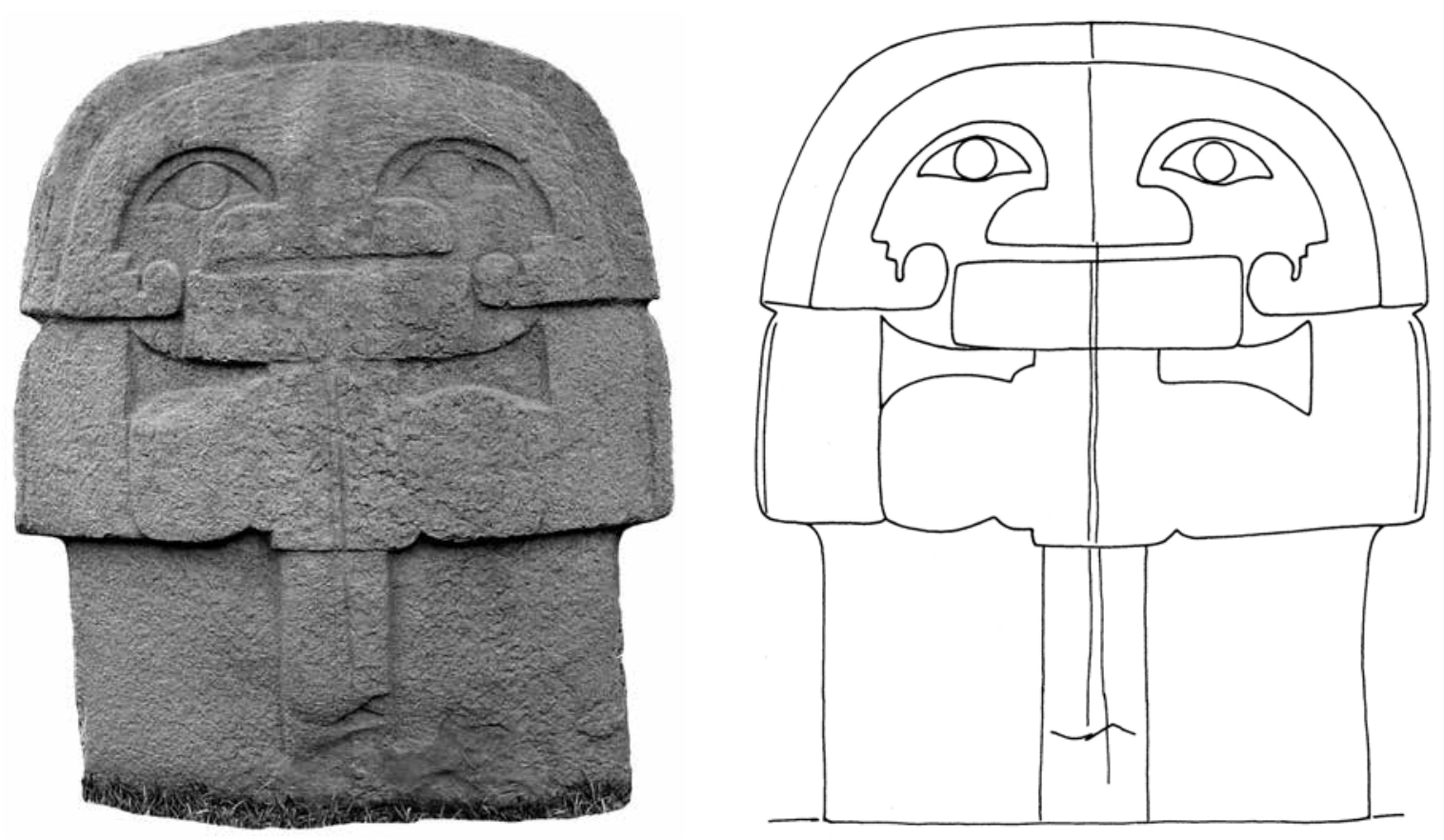

Figura 7. Escultura MC-109.

Figure 7. Sculpture MC-109.

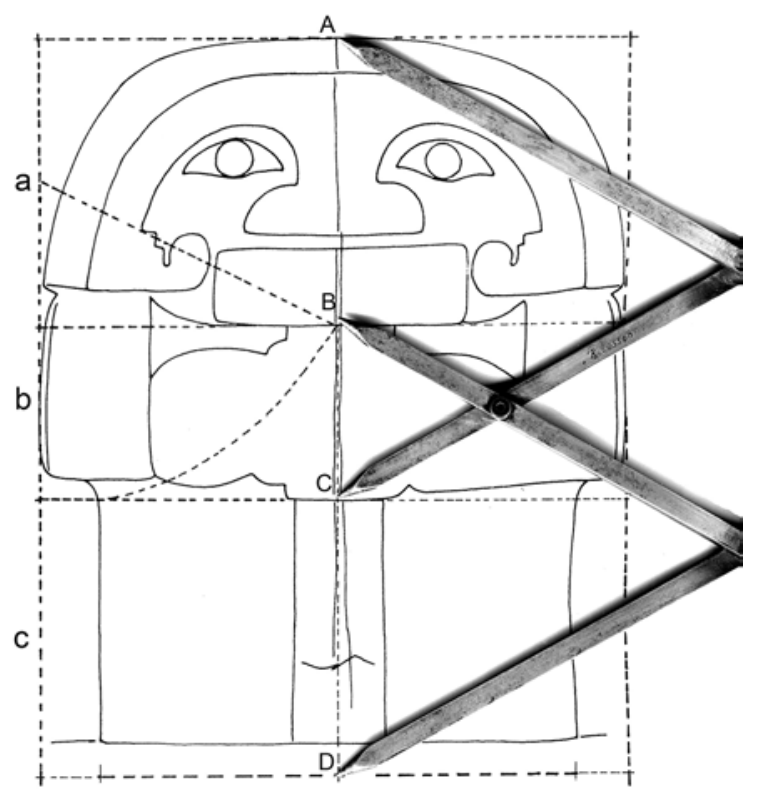

Figura 8. Aplicación del "compás áureo".

Figure 8. Application of the "golden compass."

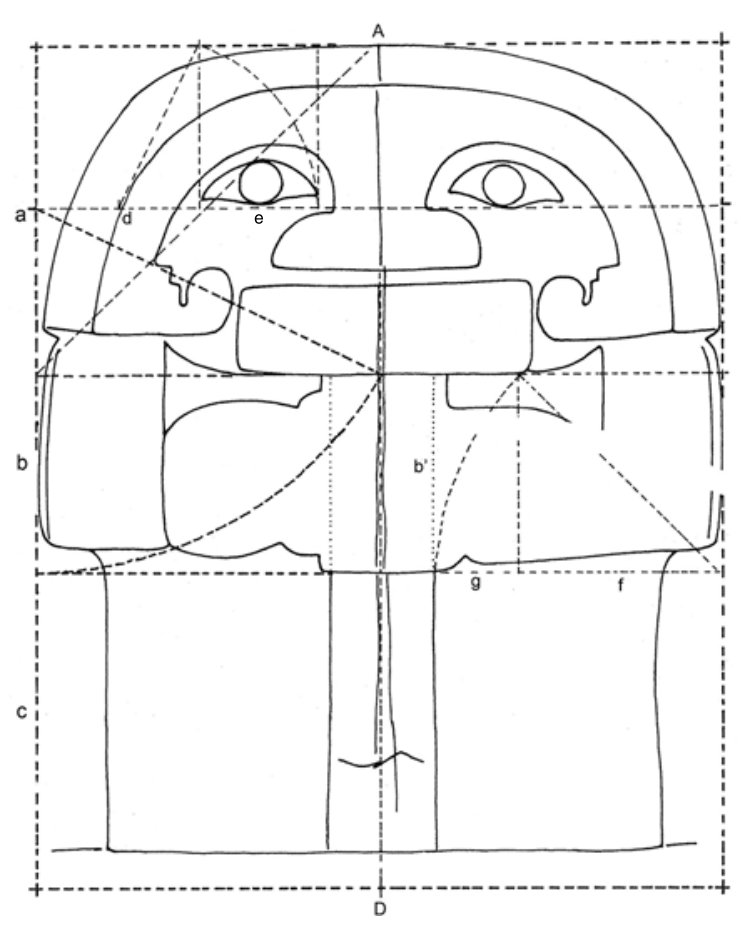

Figura 9. Escultura MC-109.

Figure 9. Sculpture MC-109. 


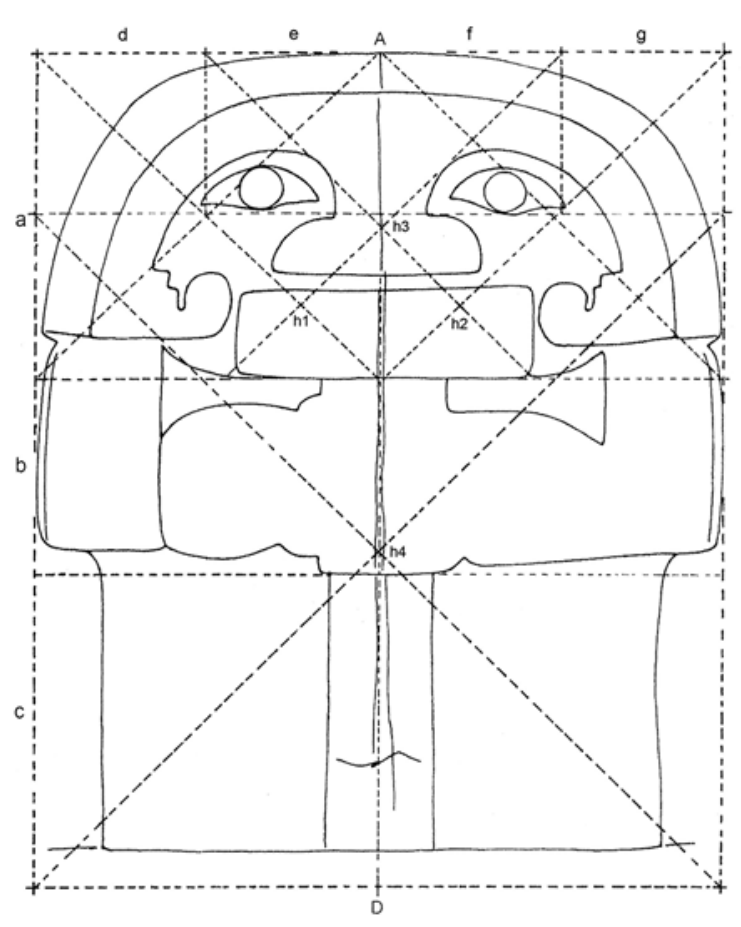

Figura 10. Escultura MC-109.

Figure 10. Sculpture MC-109.

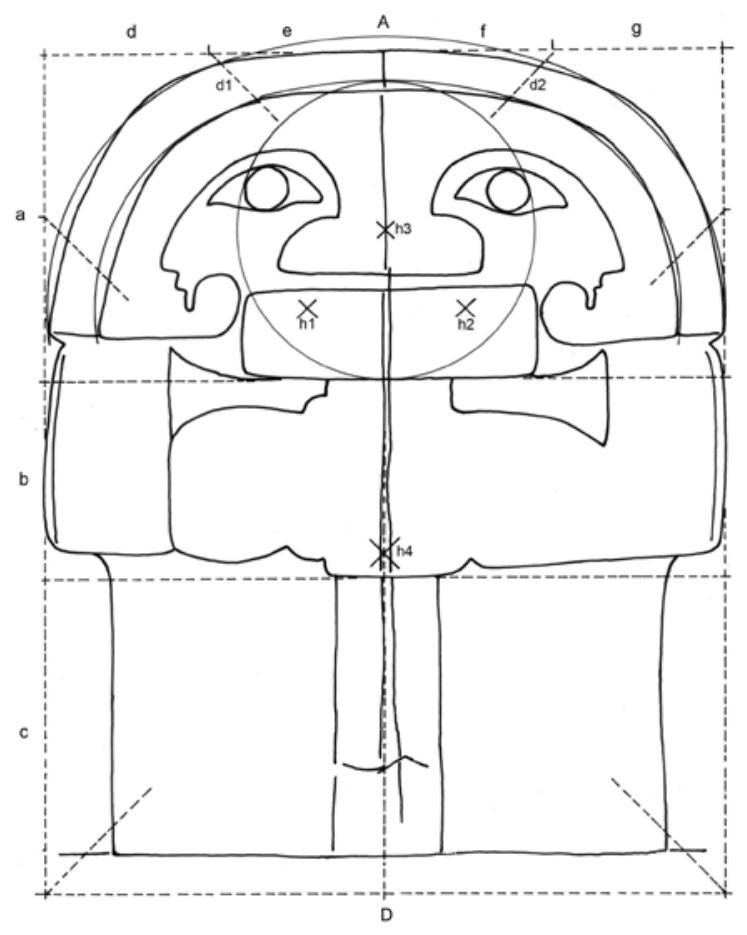

Figura 11. Escultura MC-109. Construcción de elementos. Figure 11. Sculpture MC-109, construction of elements.

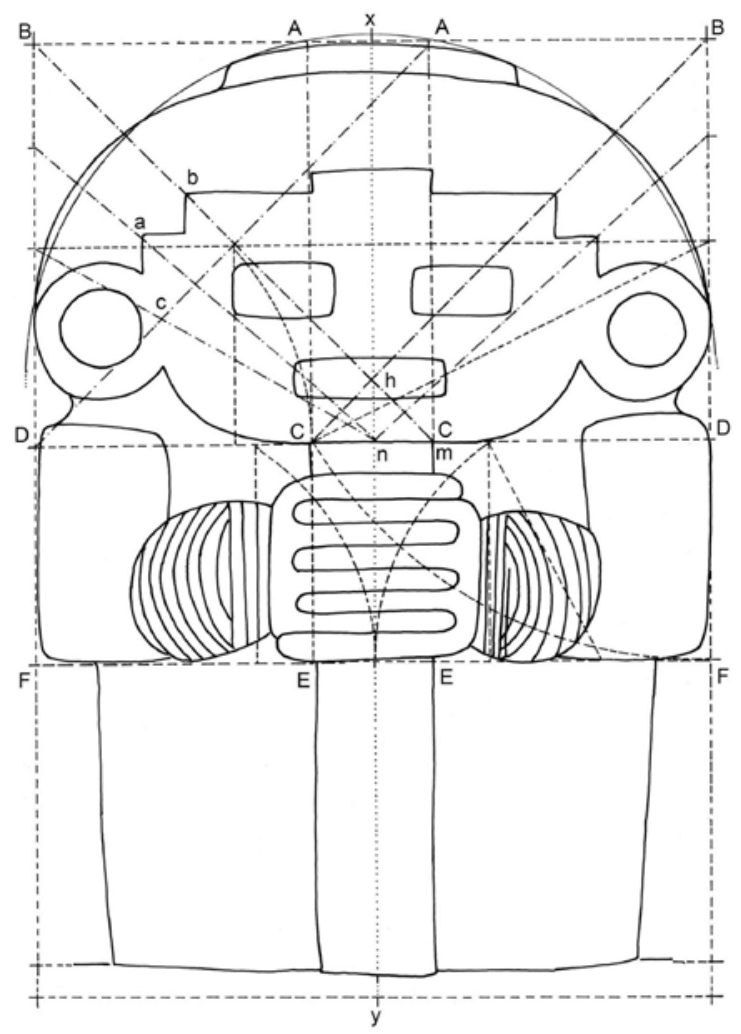

Figura 12. Escultura QB-157. Construcción de elementos. Figure 12. Sculpture QB-157, construction of elements.

fue un quebradero de cabeza a la hora de entender su composición. Al aplicar el "compás de oro" encontré que está compuesta por la superposición de dos rectángulos dorados.

De arriba hacia abajo se observa el cuadrado ABCD que genera el rectángulo CDEF para formar el rectángulo mayor ABEF. Este rectángulo se desdobla hacia el lado opuesto y genera el rectángulo complementario. Las diagonales de los cuadrados se intersecan en el punto $h$, en el centro de la boca, desde ella se traza el hemiciclo que forma la parte superior de la cabeza. Las diagonales y líneas trazadas desde los puntos medios de los lados de los cuadrados hasta el punto de intersección ( $\mathrm{n}$ ) de la línea media x y, determinan los puntos ( a b c ) para definir el diseño del tocado y las orejeras. Las manos con los dedos entrelazados se lograron mediante el mismo procedimiento de invertir los rectángulos dorados.

En la escultura QB-156 del sitio Quebradillas (fig. 13) se aplicó un procedimiento similar al anterior. El rectángulo dorado ABEF (formado por el cuadrado ABCD y el rectángulo menor $\mathrm{CDEF}$ ) se desdobla en el rectángulo abef (formado por el cuadrado abcd y el rectángulo 


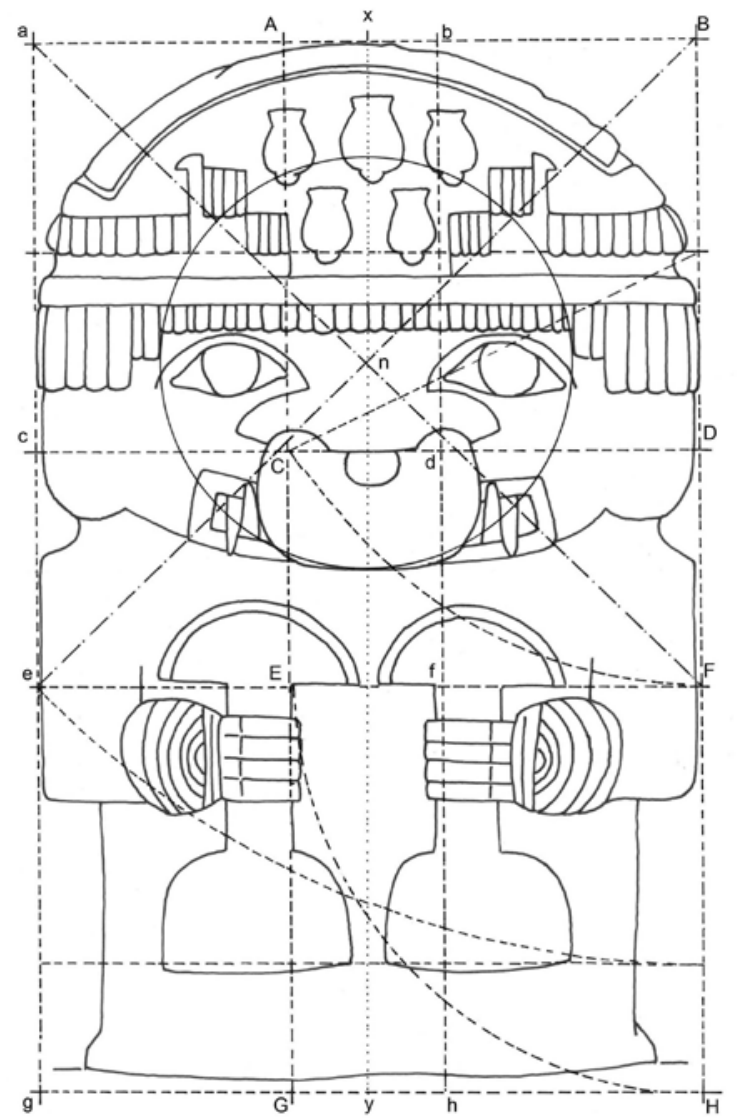

Figura 13. Escultura QB-156. Construcción de elementos. Figure 13. Sculpture QB-156, construction of elements.

menor cdef). Pero a diferencia de otros casos en que la zona inferior de la pieza está constituida por un rectángulo, en este caso está formado por dos cuadrados superpuestos que se desdoblan en rectángulos dorados hacia la zona superior de la figura. Así, el rectángulo menor CDEF es común al rectángulo dorado CDGH que, por tanto, se superpone al rectángulo dorado ABEF. Y de la misma manera ocurre con los dos rectángulos complementarios de la zona opuesta: abef se superpone a cdgh. Al trazar las diagonales aF y eB se obtiene el punto $n$, desde donde se traza el hemiciclo para definir la estructura del tocado y los diferentes puntos de referencia para diseñar la cara.

La escultura numerada como AI-272, procedente del Alto de los Ídolos, en el municipio de Isnos (fig. 14), se encuentra trabajada en la proyección formal que algunos expertos llaman como de "bulto redondo" o volumétrica, con el sentido de que se trata de una proyección tridimensional. Pero como ya he planteado en un trabajo anterior:

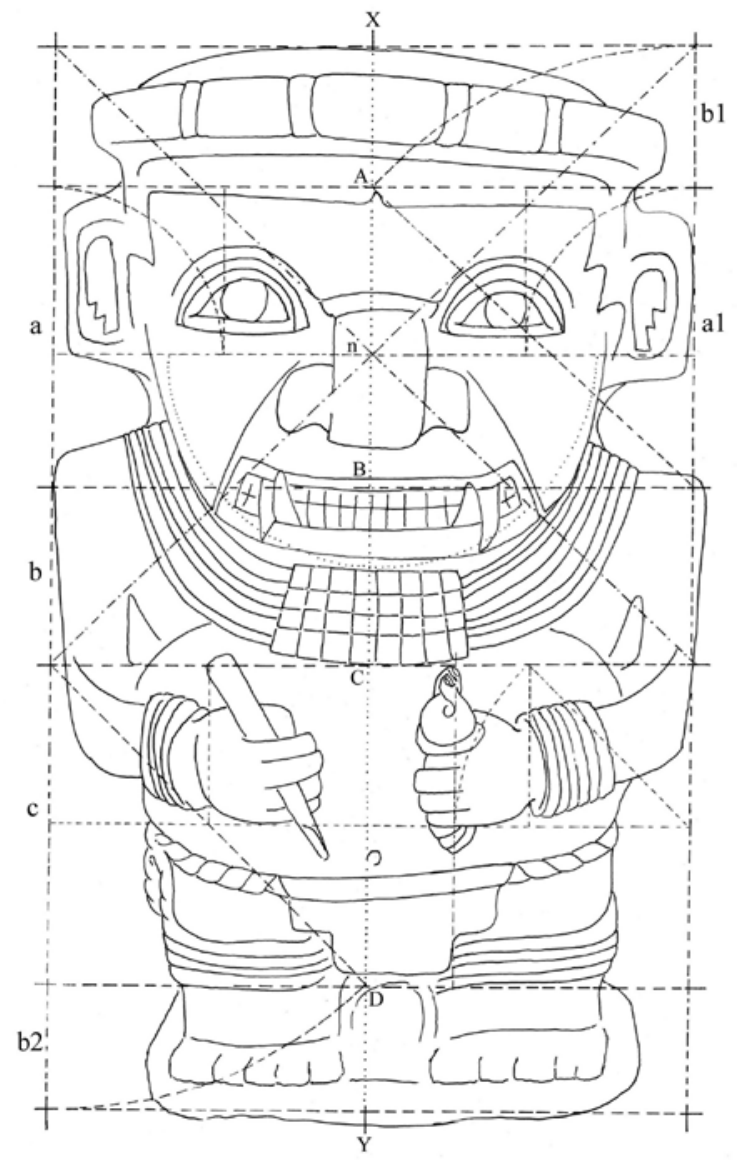

Figura 14. Escultura AI-272. Construcción de elementos. Figure 14. Sculpture AI-272, construction of elements.

Con notables excepciones, en la escultura del Clásico y de los períodos Tardíos o Recientes, la mayor parte de las representaciones trabajadas en material lítico son planigráficas o suponen un concepto planario de la representación. Desde el "monolito Bennet" en Tiahuanaco a la estatuaria de Pukará en el Titicaca y Recuay en el valle de Huaraz y Chavín de Huántar en Perú y, pasando por San Agustín en el sur de Colombia, hasta los atlantes de Tula en México, la mayor parte de la estatuaria ("redonda" o "de bulto") y de las técnicas escultóricas, ya sea en relieves y en estelas, es planigráfica. Consisten fundamentalmente en la proyección de cuatro a seis planos yuxtapuestos para conformar un aparente volumen. Este "volumen" está determinado por el volumen físico de la masa de material lítico, pero no es un "volumen escultórico" (Velandia 2006: 223).

Es decir, la proyección no es tridimensional, sino de dos dimensiones o planaria. Y este es el caso de la escultura AI-272 que, además de estar trabajada por los cuatro planos, se encontraba decorada con pintura (amarillo, blanco, rojo y negro). El procedimiento para el análisis de esta pieza consistió en la determinación, con el "compás de oro", de cuatro puntos específicos (ABCD) sobre la línea de simetría XY. En este caso, a 
diferencia de los anteriores, los rectángulos dorados no se superponen sino que coinciden exactamente sobre la línea de simetría y el lado de cada cuadrado es exactamente el complementario del opuesto. El cuadrado a1 genera el rectángulo b1 hacia arriba, para determinar el punto máximo de altura de la escultura, mientras el cuadrado c genera el rectángulo b2 hacia abajo, para marcar el punto máximo opuesto. La intersección de las diagonales del cuadrado superior, compuesto por a / a1 más b y b1, genera el punto n desde el cual se dibuja el círculo que conforma la cara de la escultura. Los demás elementos, posición de las manos, punto extremo de los ojos, ancho de la boca, etc., se determinan por el juego de cuadrados y rectángulos armónicos.

La eficacia del análisis de la proporción armónica en la estatuaria de San Agustín se puso a prueba en el 2008, cuando en el curso de una exposición de arqueología organizada por el Museo de América en Madrid, en homenaje a José Pérez de Barradas, en que se describía su aporte a la arqueología americana y en particular sus trabajos en San Agustín, encontré una pequeña escultura descrita con la etiqueta: "Figura antropomorfa, Cultura de San Agustín (Colombia), 1000 AC-1500 DC". Con el apoyo del Dr. Félix Jiménez Villalba, subdirector del Museo de América, quien gentilmente me facilitó el acceso directo a la pieza, pude realizar una detenida observación, fotografías y dibujos pertinentes (figs. 15 y 16).

Desde la primera observación en la vitrina de la exposición consideré que la escultura era falsa, pues aparte de algunos elementos desconocidos en la estatuaria agustiniana, como los pequeños discos en la frente sobre el borde del casco y el remate sobre la cabeza, lo que me parecía discordante era la presencia del pez sostenido con ambas manos sobre el vientre, pues en San Agustín, hasta ahora, era una rareza la repetición de un tema como en el caso de las representaciones de un águila sosteniendo una serpiente entre el pico y las patas (esculturas MB-022 de la Mesita B y escultura CP-163 del Cerro de la Pelota), y esta pieza del Museo
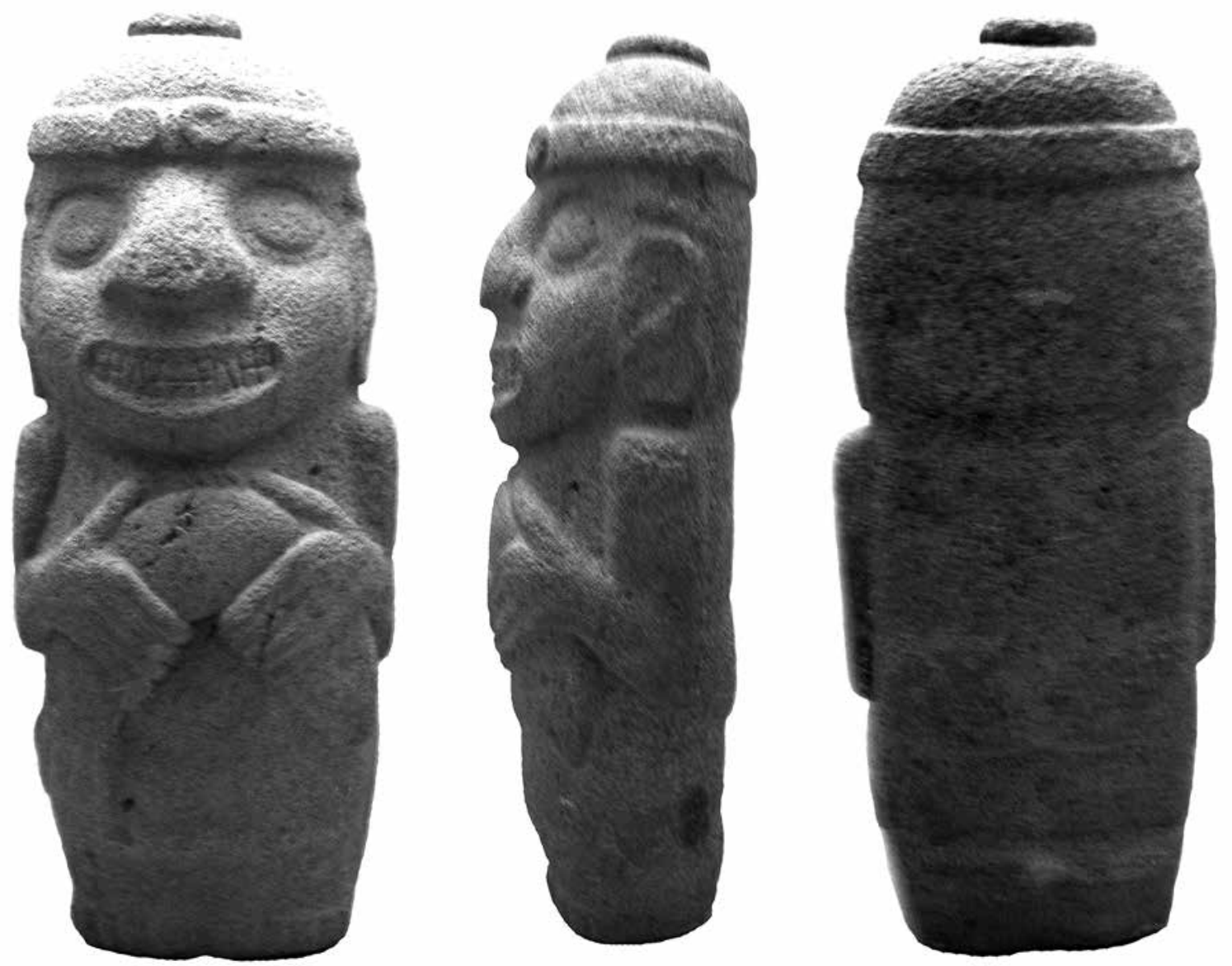

Figura 15. Escultura PD-461-A.

Figure 15. Sculpture PD-461-A. 

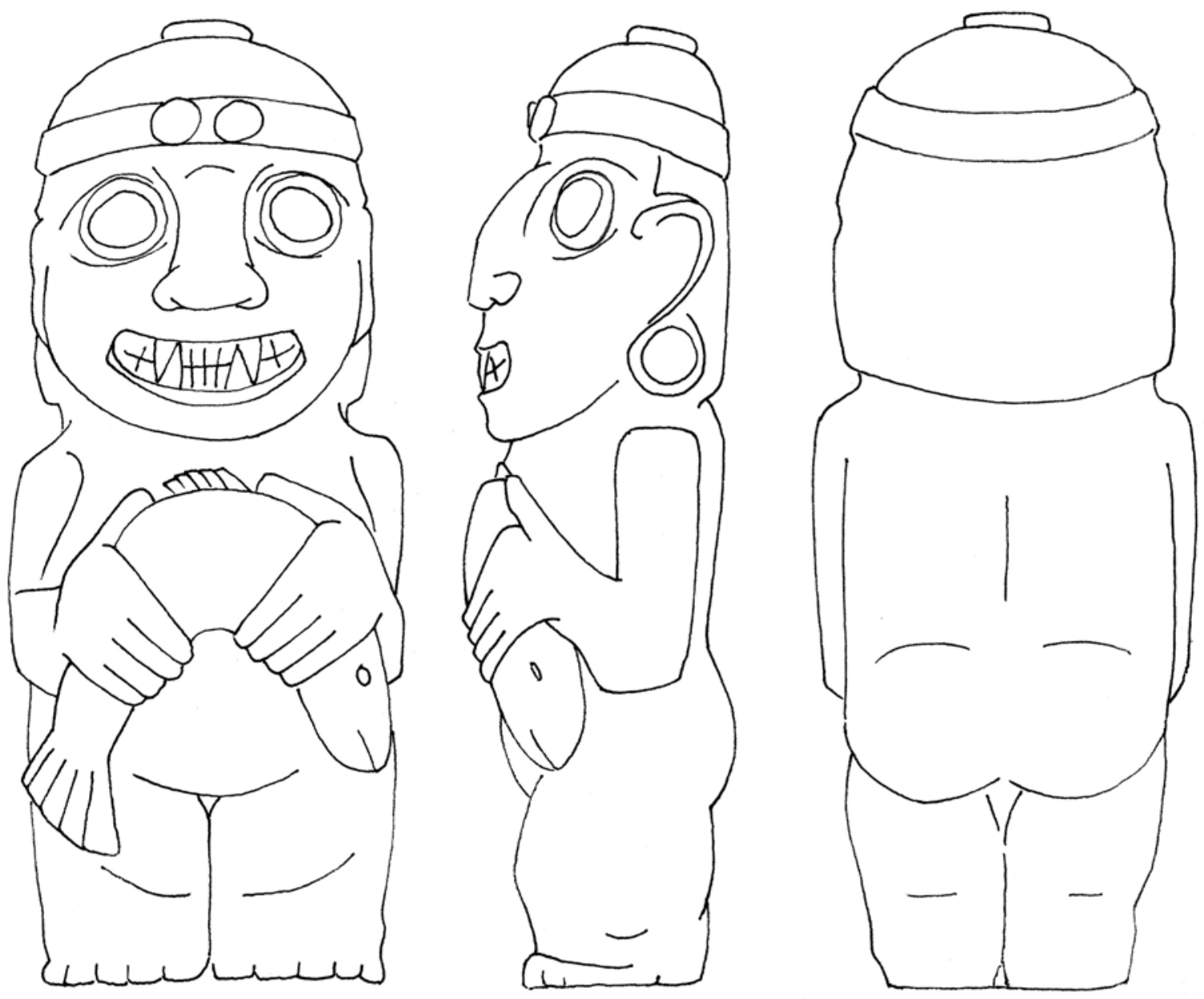

Figura 16. Escultura PD-461-A.

Figure 16. Sculpture PD-461-A.

de América repetía, de manera casi idéntica, una pieza de la Mesita B, la número MB-041.

La observación del estado de la pátina, el tratamiento técnico aplicado mediante cincelado y abrasión, y algunos elementos iconográficos, como el diseño de la espalda con la distinción natural de las nalgas y el diseño de las orejas y la boca, así como la proporción entre la cabeza, el cuerpo y las manos, me fueron llevando a cambiar de criterio. El concepto que ya he planteado acerca de la estructura de la estatuaria y la proporción armónica implica una situación: que los artesanos copistas, o "falsificadores" según el caso, remedan la forma pero no la estructura; es decir, pueden copiar el "parecido" formal pero no pueden reconstruir la estructura, porque no la pueden comprender, ya que hace parte de una noción estética diferente a su patrón cultural estético.

Para resolver las objeciones y las dudas acerca de su originalidad, solo quedaba verificar si estaba diseñada de acuerdo con el concepto de la proporción armónica, mediante la aplicación del compás d’or (fig. 17).

El compás áureo permitió definir sobre la línea de simetría tres puntos, de arriba hacia abajo, x, y, z, estos marcan la relación entre el lado (ab) de un rectángulo (xayb) y el lado (bc) de un cuadrado (ybxc) que, articulados, conforman un rectángulo cuyos componentes (rectángulo y cuadrado) se encuentran en una relación armónica. Esta misma relación se repite dos veces a lo largo de la línea de simetría, hasta la base de la escultura. El punto (n), ubicado en el punto medio de la base del cuadrado (ybxc), es el centro desde el cual se traza un círculo que define el segmento de círculo para delimitar la cabeza y formar el contorno del casco, en tanto que describe la forma y los límites de la cara. Todos los demás puntos para definir las manos y el pez están rigurosamente articulados dentro de este juego de relaciones espaciales. La conclusión es que la escultura 


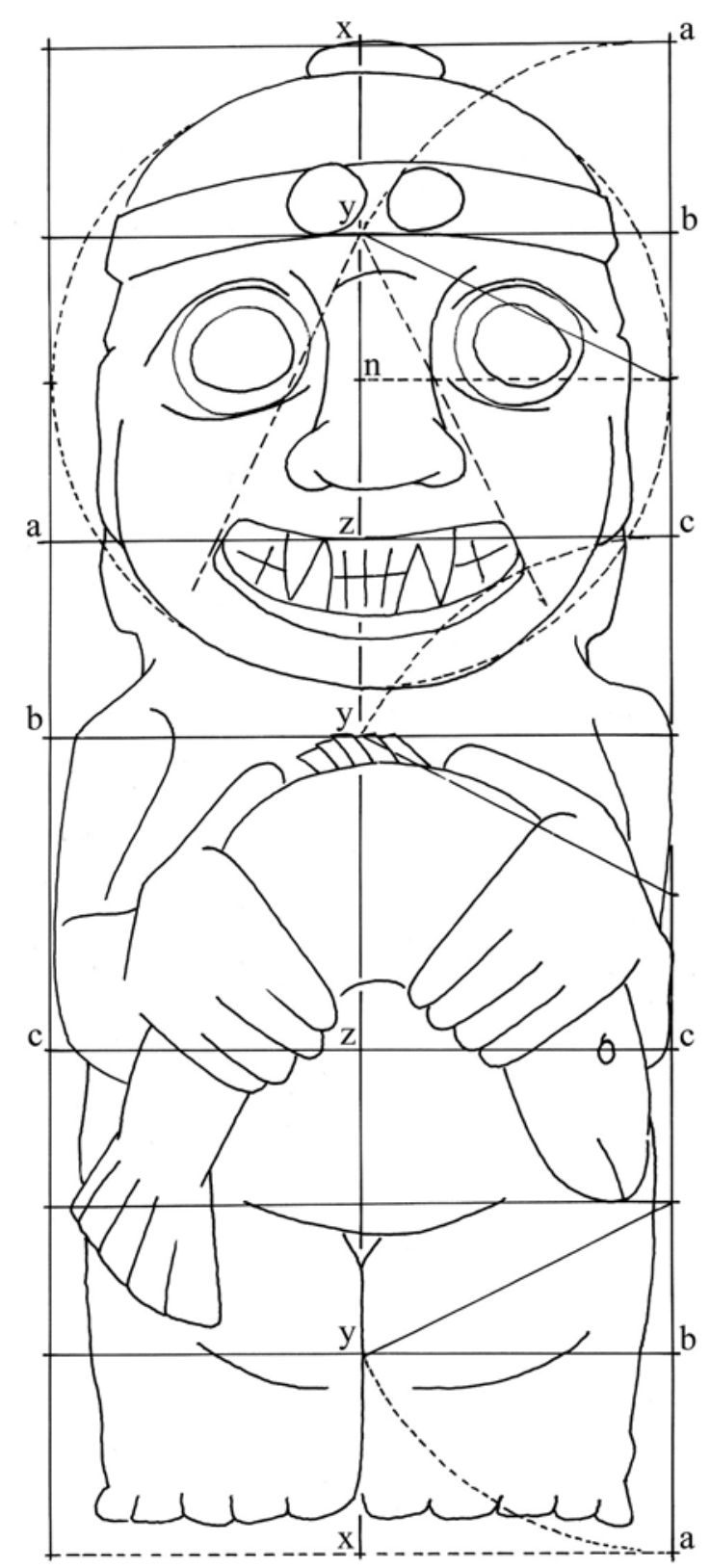

Figura 17. Escultura PD-461-A.

Figure 17. Sculpture PD-461-A.

no pudo ser hecha por persona distinta a un portador de una noción estética específica, la de la cultura de San Agustín. La pieza, por tanto, es original.

\section{CONSIDERACIONES FINALES}

He mostrado en un grupo de piezas, elegidas por ser significativas de la estatuaria de San Agustín, el proceso de diseño de las representaciones plásticas, el que se ciñe a un canon específico, cuyo fundamento es la proporción armónica. En algunos casos la norma no funciona de manera tan estricta como en los casos seleccionados para esta explicación, pero aunque el canon fuera aplicado de manera heterodoxa -como en ciertas esculturas atribuidas al Período Formativo-, de todas formas se conserva el concepto de la proporción armónica.

Como toda propuesta de solución a un problema, otras preguntas se vuelven más estrictas y por tanto se generan nuevos problemas. Entre las más afinadas, tenemos la de cómo procederían los artesanos lapidarios al diseño de cada pieza, pues aparte de algunos supuestos "borradores" ejecutados en pequeñas láminas de piedra, no existe en el registro de San Agustín ningún boceto previo del cual se pudiera deducir o, en el caso que me ocupa, demostrar el modo de aplicación de un proceso fundamentado en diseños armónicos. Se conocen 17 piezas que no fueron terminadas, ya que el trabajo se suspendió en distintos momentos de su realización; pese a que en ellas se reconoce la proporción armónica, tampoco permiten inferir el planteamiento inicial para el diseño, es decir, un boceto o una proyección del trabajo a ejecutar.

Otras preguntas salen de las mismas dificultades para el análisis geométrico, pues los artesanos de San Agustín, aunque seguían ordenamientos específicos y directrices precisas en cuanto al diseño formal, podrían producir variaciones atribuibles a la relativa habilidad en el manejo de los instrumentos -que, además, aún no conocemos- y a la diversidad de los materiales con que podían contar. Todo esto determina que las mediciones no son tan precisas y que en algunos casos -como en la escultura AI-272, analizada- el cuerpo se desplaza hacia un costado. De otra parte, las rocas andesíticas y tobas volcánicas, que fueron la base para la elaboración escultórica, no son uniformemente compactas y esto determina dificultades prácticas a la hora de desbastar, perforar, cortar o cincelar sobre un patrón de diseño específico.

La aplicación del concepto geométrico de la proporción armónica en América es más extensa de lo que parece a primera vista, pues aunque son escasos los trabajos de investigación -y numerosas las especulaciones esotéricas-, los casos conocidos, tanto en Mesoamérica como en el área sudamericana, son suficientes para presumir que corresponden a unos saberes más extensamente compartidos por distintos pueblos prehispánicos. La denominada "greca" escalonada, por ejemplo, en sus múltiples variaciones, que se encuentra en muchas culturas de Sudamérica y del área mesoamericana, constituye 
un referente importante al momento de considerar la dispersión relativa de este rasgo cultural y de los modos de aplicación de un concepto estético como la proporción armónica. Igual cosa ocurre con los diseños de la orfebrería en oro, tumbaga y cobre, de las culturas prehispánicas del Área Intermedia (Colombia, Ecuador, Venezuela y Costa Rica) y desde allí hasta Mesoamérica.

De todas maneras, solo la futura investigación arqueológica nos permitirá dilucidar y ampliar el alcance de estos debates y propuestas de interpretación y, en este sentido, el presente trabajo pretende contribuir a la reformulación de las preguntas de investigación. Aunque no siempre se encuentra lo que se anda buscando, el trabajo produce cosas más concretas cuando sabemos lo que buscamos.

RECONOCIMIENTOS La terminación de este trabajo tuvo la subvención de una estancia en el Instituto de Ciencias del Patrimonio (Incipit) del Consejo Superior de Investigaciones Científicas (CSIC), en Santiago de Compostela, bajo la dirección de Felipe Criado Boado y con el apoyo de Juan Manuel Vicent García y Manuel Santos Estévez en el Departamento de Prehistoria del Centro de Ciencias Humanas y Sociales del CSIC, en Madrid; a quienes expreso mi reconocimiento y gratitud. De igual manera, agradezco la generosidad de M. Robert Losson, en Auvillar, Francia, al facilitarme la herramienta decisiva para resolver el problema que justifica este trabajo.

\section{NOTAS}

${ }^{1}$ A partir de la teoría sobre la proporción armónica en las artes y la naturaleza, de Matila Ghyka (1953) y de su propia experiencia en los museos y con las colecciones arqueológicas de México, Rengifo propuso un procedimiento analítico de la estatuaria, mediante el cual demostró que la descripción estética de los artefactos arqueológicos, en especial cuando estos rebasaban su función puramente utilitaria, era una alternativa explicativa para la arqueología; tesis que, a decir verdad, fue recibida con cierta indulgencia por los arqueólogos. En las tres décadas siguientes esta propuesta analítica (el estudio estético de las estructuras en el registro arqueológico) se convirtió en una veta de trabajo que abarca desde el estudio de las artes plásticas, la cerámica y los textiles hasta el arte rupestre. Los trabajos acerca de la lógica de las representaciones simétricas (Washburn \& Crowe 1988; Kubovy \& Strother 2004), el arte mural (Westheim 1957), la estatuaria (Williams 1992; Martínez del Sobral 2000), la arquitectura (Kubler 1986), etc., produjeron un ingente aporte a la mejor comprensión de la arqueología americana.

2 De la Fuente (1992), Williams (1992), Jiménez (1998), Albarrán (2001). En rigor, son muy pocos los estudios iconográficos desde este punto de vista teórico.

3 Alta France Productions, Robert Losson, 82340, Auvillar, Francia, <www.robertlosson.com/fr/alta5.htm> [Citado: 16-04-2015]

${ }^{4}$ Algunas esculturas tienen, bajo la línea inferior de la superficie esculpida, una especie de peana o pedestal que oscila entre 10 y 25 centímetros de altura, de manera que, en estos casos, dicho espacio hace parte de la distribución espacial en el diseño ortogonal. Pero en la mayor parte de las esculturas -incluidas las especificadas-, en la zona inferior -que por razones museográficas no se muestra-, ostentan una especie de peonza o extremo triangular que en algunos casos llega a ser la tercera parte del total de la estatua, y que sirve para desplazar hacia abajo el centro de gravedad de la pieza, de tal manera que esta se mantiene erecta, en la forma como aparecen en los recintos funerarios o dólmenes, que constituyen el núcleo del montículo megalítico. Estas prolongaciones del material de base de la escultura no hacen parte de la composición escultórica y, por tanto, quedan por fuera del diseño de la proporcionalidad armónica.

\section{REFERENCIAS}

Albarrán, A., 2001. Estudio geométrico de un fragmento del mural de las "Diosas verdes" de Tetitla. La pintura mural prebispánica en México, Boletín informativo año VII, $\mathrm{N}^{\circ}$ 15: 12-22. México, D. F.: Universidad Nacional Autónoma de México, Instituto de Investigaciones Estéticas.

De la Fuente, B., 1992. Cabezas colosales olmecas. México, D. F: El Colegio Nacional.

CORBALÁn, F., 2009. La proporción áurea, el diseño y la naturaleza. En La proporción: Arte y matemáticas, J. Giménez, Coord., pp. 103-121. Barcelona: Editorial Graó.

Gнука, M., 1953. Estética de las proporciones en la naturaleza y en las artes. Buenos Aires: Editorial Poseidón.

Hemenway, P., 2008. Divine Proportion. $\phi$ Phi in Art, Nature and Science. Lugano: Springwood S. A.

Jiménez, E., 1998. Iconografía de Tula. El caso de la escultura. México, D. F: Instituto Nacional de Antropología e Historia.

Kubovy, M. \& L. Strother, 2004. The Perception of Band Patterns. Going beyond geometry. En Embedded Symmetries: Natural and cultural, D. Washburn, Ed., pp. 19-26. Albuquerque: University of New Mexico Press.

Kubler, G., 1986. Arte y arquitectura en la América precolonial. Madrid: Editorial Cátedra.

Llanos, H., 1995. Los chamanes jaguares de San Agustín: Génesis de un pensamiento mitopoético. Bogotá: Edición H. Llanos.

MARTínez Del Sobral, M., 2000. Geometría mesoamericana. México, D. F.: Fondo de Cultura Económica.

Orozpe, M., 2010. El código oculto de la greca escalonada. México, D. F.: Universidad Nacional Autónoma de México.

PACiOli Di Borgo, L., 1991 [1509]. La divina proporción. Madrid: Ediciones Akal.

Rengifo, L. A., 1962. El águila monolítica agustiniana. Bogotá: Universidad Nacional de Colombia, Escuela de Bellas Artes.

RENGIFO, L. A, 1966. La proporción armónica en la estatuaria agustiniana. Bogotá: Universidad Nacional de Colombia, Escuela de Bellas Artes.

RODríguez, C. A., 2005. Los hombres y las culturas prehispánicas del suroccidente de Colombia y el norte del Ecuador. Cali: Universidad del Valle, Editorial de la Facultad de Humanidades.

SAMPAOLESI, R., 2006. La divina proporción y la retina. Buenos Aires: Olmo Ediciones.

Velandia, C., 1994. San Agustín. Arte, estructura y arqueología. Modelo para una semiótica de la iconografía precolombina. Bogotá: Fondo de Promoción de la Cultura, Banco Popular \& Universidad del Tolima.

Velandia, C., 1999. The Archaeological Culture of San Agustín. Towards a New Interpretation. En Archaeology in Latin America, G. Politis \& B. Alberti, Eds., Chapter 10, pp.185-215. London: Routledge, Taylor \& Francis Group.

Velandia, C., 2006. Prolegómenos a la construcción de una semasiología prehispánica. En Arqueología Suramericana 2 (2): 205-243, Catamarca.

Washburn, D. \& D. Crowe, 1988. Symmetries of Culture. Theory and practice of plane pattern analysis. Seattle: University of Washington Press.

WeIL, H., 1990. Simetría. Madrid: Editorial McGraw-Hill.

Westheim, P., 1957. Ideas fundamentales del arte prebispánico en México. México, D. F.: Fondo de Cultura Económica.

Williams, E., 1992. Las piedras sagradas. Escultura prebispánica del occidente de México. Zamora: El Colegio de Michoacán. 